\title{
A Case of Escherichia coli Endocarditis After Hemorrhoidectomy Performed by a Herbalist
}

\author{
Ahmet Can Şenel, Joanna Ondrush \\ Department of Anesthesiology and Critical Care, George Washington University Medical Center, Washington DC, USA
}

\begin{abstract}
We describe the first reported case of Escherichia coli endocarditis following a hemorrhoidectomy that was performed by the patient's herbalist. With increasing frequency, patients are seeking care by those who practice alternative medicine. Physicians must become more aware of the impact and possible complications related to this practice. Prosthetic valve endocarditis ranges up to $1.5 \%$ per patient-year for mechanical mitral valves and up to $0.1 \%$ per patient-year for other valves. Although the incidence of aortic prosthetic valve endocarditis is not uncommon, the concomitant organism and its mode of bacteremia make this a most unusual case. There is a paucity of information in the literature regarding surgical complications performed by alternative medicine practitioners. To our knowledge, a case of gram-negative endocarditis as a result of hemorrhoidectomy performed by an herbalist has never been reported.
\end{abstract}

Key Words: Endocarditis, Escherichia coli, hemorrhoidectomy, alternative medicine, complications

\section{Case Report}

The patient is a 60 year old man with a St. Jude aortic valve for a bicuspid valve placed seven years prior to admission. He went to see his herbalist for treatment of hemorrhoids, which were subsequently removed by an unknown technique. Three days after this unknown technique, the patient developed fever and chills. He was admitted to another hospital, at which time blood cultures grew pen sensitive Escherichia coli and a negative abdominal CT scan. He was treated with one week of IV ampicillin/sulbactam followed by two additional weeks of oral amoxicillin/clavulanic acid. He had been symptom free during this period. Upon cessation of the antibiotics, he again developed fever and chills, in addition to nausea, diarrhea and abdominal pain. The patient was then admitted to the George Washington University Hospital (Washington D.C.) for further work-up.

On physical examination, the patient was alert and oriented, but toxic and pale in appearance. Blood pressure was $90 / 62 \mathrm{~mm} / \mathrm{Hg}$, heart rate 111 beats $/ \mathrm{min}$. respiratory rate was 16 breaths $/ \mathrm{min}$. and temperature was $37.8^{\circ} \mathrm{C}$. His neck was supple without JVD. The cardiac exam showed a regular rate and rhythm with a systolic ejection murmur and an audible mechanical click from the artificial valve. Lungs were clear to auscultation bilaterally. There was mild tenderness in the bilateral lower abdominal quadrants. Rectal examination showed external hemorrhoids with an area of excoriation approximately $0.5 \mathrm{~cm}$ wide where the previous hemorrhoid had been removed. There was no pedal oedema. Admission serum che- mistry values were as follows: sodium $130 \mathrm{mmol} / \mathrm{L}$; potassium $4.0 \mathrm{mmol} / \mathrm{L}$; chloride $97 \mathrm{mmol} / \mathrm{L}$; carbon dioxide $22 \mathrm{mmol} / \mathrm{L}$; urea nitrogen $6 \mathrm{mmol} / \mathrm{L}$, creatinine $0.6 \mathrm{mg} / \mathrm{dL}$; glucose 117 $\mathrm{mg} / \mathrm{dL}$; the initial $\mathrm{CBC}$ showed a white count of $7.000 / \mathrm{mm}^{3}$, $\mathrm{Hg} 10 \mathrm{~g} / \mathrm{dL}$, Htc $30 \%$, platelet count $285.000 \mathrm{~mm}^{3}$ with a differential of $78 \%$ segmented neutrophils and $14 \%$ lymphocytes. Cardiac enzymes were normal. Chest $X$-ray was normal. Electrocardiogram showed a new first degree AV block. In addition, patient tested positive for Clostridium difficile toxin in his stool. During this hospitalization the patient was started on ampicillin/sulbactam $3 \mathrm{gm} \mathrm{IV} \mathrm{q6} \mathrm{and} \mathrm{gentamicin} 90 \mathrm{gm} \mathrm{IV} \mathrm{q8.}$ Blood cultures from admission grew pan-sensitive Escherichia coli and Citrobacter amalonaticus. A transthoracic echo was negative but a transesophageal echo showed vegetations on the prosthetic aortic valve with ring abscess. The cardiothoracic surgery department was consulted. Within 24 hours the patient went into septic shock and was deemed to unstable for surgery.

Within 48 hours his hemodynamics stabilized, but his ECG had progressed to a junctional rhythm with arrhythmic AV dissociation. He was taken to the operating room where an excision of the prosthetic valve and drainage of the abscess were carried out. A new St. Jude valve was placed. Pathology showed acute and organizing fibrinoprulent exudates consistent with endocarditis. Culture from the aortic valve grew Escherichia coli. The patient did well post-operatively and was discharged home with an additional five weeks of IV antibiotics. 


\section{Discussion}

There have been numerous studies evaluating the risk factor development of prosthetic valve endocarditis (1). Calderwood, et al. analyzed the outcome of 116 patients with prosthetic valve endocarditis $(2,3)$ and concluded that aortic valve infection and the onset of endocarditis within 12 months of the cardiac operation were risk factors for complicated endocarditis (4). Depending on the study, the incidence of gramnegative endocarditis ranges from 2-10\% (5). Gram negative endocarditis, although less common, does not seem to pose an increase risk in mortality when it is the infecting organism. Most studies, when defining the infecting organism, group all gram-negative rods together in their analysis. We found isolated case reports of endocarditis caused by $E$. coli (6). In our patient, E. coli grew from the valve $(7,8)$. The onset of his symptoms correlates with the office procedure that was performed, and although the patient did not know the details of how the hemorrhoid was removed, he stated that no antibiotic prophylaxis was given. There are clear guidelines as to the indication for antibiotic prophylaxis in patients with prosthetic valves $(9,10)$. For colorectal surgery, antibiotics should be given prior to incision and continued 24 hours after surgery, however, a gold standard for an antibiotic regimen has never been clearly identified (11). As for gastrointestinal endoscopic procedures, specifically colonoscopy with or without biopsy/ polypectomy, there is insufficient data to make firm recommendations, as this procedure is associated with a low risk of bacteremia (12). Our patient, like many patients, was seeking complementary or alternative forms of medicine, often without the knowledge of their physician. Drug interaction and toxicities from the use of alternative medicines have been increasingly documented in the literature. One study looked at the use of alternative medicine by surgical patients and found that $27 \%$ of the consumed products may inhibit coagulation, as well as other potential adverse effects such as sedation, cardiac and electrolyte abnormalities (13). Few studies have addressed patients who sought surgical procedures from alternative medicine practitioners. We found one case report of a patient who developed life threatening perineal gangrene from rectal perforation following colonic hydrotherapy (14). Part of the danger lies not only in the procedures themselves, but also in the lack of communication between physician and patient that these alternative therapies are being pursued.
With increasing knowledge we can help bridge the gap and decrease the risk to patients.

\section{Conflict of Interest}

No conflict of interest was declared by the authors.

\section{References}

1. Vlessis AA, Khaki A, Grunkemeier GL, Li HH, Starr A. Risk, diagnosis and management of prosthetic valve endocarditis: a review. J Heart Valve Disease 1997;6:443-65.

2. Arvay A, Lengyel M. Incidence and risk factors of prosthetic valve endocardits: Eur J Cardiothrorac Surg 1988;2:340-6.

3. Calderwood SB, Swinski LA, Karchmer AW, Waternaux CM, Buckley MJ. Prosthetic valve endocarditis. Analysis of factors affecting outcome of therapy. J Thorac Cardiovasc Surg 1986;92:776-83.

4. Mansur AJ, Dal Bo CM, Fukushima JT, Issa VS, Grinberg M, Pomerantzeff PM. Relapses recurences, valve replacements and mortality during the long term follow-up after infective endocarditis American Heart J 2001;141:78-86. [CrossRef]

5. Wolff M, Witchitz S, Chanstang C, Regnier B, Vachon F. Prosthetic valve endocarditis in the ICU. Prognostic factors of overall survival in a series of 122 cases and consequences for treatment decision. Chest 1995;108:688-94. [CrossRef]

6. Mylonakis E, Calderwood SB. Infective endocarditis in adults. New Eng J med 2001;345:1318-30. [CrossRef]

7. Tellez I, Chrysant GS, Omer I, Dismukes WE. Citrobacter diversus endocarditis. Amer J Med Sci 2000;320:408-10. [CrossRef]

8. Clemente GC, Ruiz AJ, Vilert GE, and Garcia BF. Citrobacter freundii enddocarditis. An Med Interna 1999;16:363-4.

9. Tang R, Chen HH, Wang YL, Changchien CR, Chen JS, Hsu KC, et.al. JY. Risk factors for surgical site infection after elective resection of the colon and rectum: a single-center prospective study of 2809 consecutive patients. Ann Surg 2001;234:181-9. [CrossRef]

10. Song F, GlennyAM. Antimicrobial prophylaxis in colorectal surgery: a systematic review of randomized controlled rials. BR J Surg 1998;85:1232-41. [CrossRef]

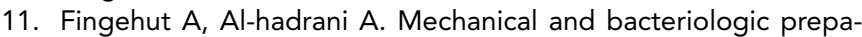
ration for colorectal surgery: Evolution and current recommendations. Associatinos of research in surgery. J Chir (paris) 1999;136:216-20.

12. Infection control during gastrointestinal endoscopy: guidelines for clinical application. From the ASGE. American Society for Gastrointestinal Endoscopy. Gastrointest Endo 1999;49:836-41. [CrossRef]

13. Norred CL, Zamudio S, Palmer SK. Use of complementary and alternative medicines by surgical patients. AANA J 2000;68:13-8.

14. Tan MP, Cheong DM. Life-threateing perineal gangrene from rectal perforation following colonic hydrotherapy: a case report. Ann Acad Med Singapure 1999;28:583-5. 